\title{
Correction to: Analytical approaches to space- and time-fractional coupled Burgers' equations
}

\author{
HODA F AHMED, M S M BAHGAT* and MOFIDA ZAKI \\ Mathematics Department, Faculty of Science, Minia University, El-Minia, Egypt \\ *Corresponding author. E-mail: msmbahgat66@ hotmail.com \\ published online 9 July 2020
}

In the recently published paper [Pramana - J. Phys. 92:8 (2019), DOI: https://doi.org/10.1007/ s12043-018-1693-z], the affiliation of the authors was inaccurate. The correct affiliation is provided here. 